УДК 536.5: 536.6: 628.8

\title{
ПОВЫШЕНИЕ ТОЧНОСТИ ИЗМЕРЕНИЯ ПЛОТНОСТИ ТЕПЛОВОГО ПОТОКА ПРИ КОНТРОЛЕ ТЕПЛОПОТЕРЬ
}

Ковтун С.И., канд. техн. наук

Институт технической теплофизики НАН Украины, ул. Желябова, 2а, Киев, 03680, Украина

У статті розглянуто аналітичні задачі, які описують найбільш типові випадки застосування перетворювачів теплового потоку для контролю тепловтрат на об'єктах різних типів. Надано рекомендації щодо підвищення точності вимірювання поверхневої густини теплового потоку в залежності від умов теплообміну та характеристик об'єкту контролю.
В статье рассмотрены аналитические задачи, которые описывают наиболее типичные случаи применения преобразователей теплового потока для контроля теплопотерь на объектах различных типов. Даны рекомендации по повышению точности измерения поверхностной плотности теплового потока в зависимости от условий теплообмена и характеристик объекта контроля.
The article is focused the analytical tasks that describe the most typical cases of using of the heat flow sensor to control the heat losses on various types objects. Recommendations to improve the accuracy of measurement of surface heat flow density depending on the heat transfer characteristics of an object-to-be-controlled.

Библ. 9, рис. 4.

Ключевые слова: плотность теплового потока, контроль теплопотерь, точность измерения.

$\mathrm{Bi}$ - критерий Био;

$D$ - диаметр, м;

$h$ - толщина, м;

$q$ - плотность теплового потока, Вт/м²;

$R$ - тепловое сопротивление, $\mathrm{M}^{2} \cdot \mathrm{K} / \mathrm{B}$;

$\mathrm{r}$ - радиус, м;

$T$ - температура, К;

При контроле теплопотерь одним из определяющих параметров является поверхностная плотность теплового потока, для измерения которой применяют те или иные преобразователи теплового потока (далее - ПТП) [1-3]. В связи с тем, что ПТП вида вспомогательной стенки, разрабатываемые в ИТТФ НАН Украины, имеют собственное тепловое сопротивление, применение их на объектах контроля различных типов при различных условиях теплообмена вносит возмущение в поля температуры и теплового потока как внутри объекта контроля, так и в самом ПТП, что неизбежно приводит к увеличению методической погрешности измерения.

Для обеспечения требуемой точности измерения поверхностной плотности теплового потока при обследовании конкретного объекта контроля необходимо применять ПТП с соответствующими параметрами: габаритными размерами, соот- $z$ - координата;

$\alpha$ - коэффициент теплообмена, Вт/ $\mathrm{M}^{2} \cdot \mathrm{K}$;

$\lambda$ - теплопроводность, Вт/м·К;

$\rho$ - приведенный радиус

ПТП - преобразователь теплового потока;

ОК - объект контроля;

ЧЭ - чувствительный элемент.

ношением размеров ПТП и его чувствительного элемента, шириной охранной зоны, теплофизическими свойствами, при которых будет обеспечена минимальная методическая погрешность. Ширина охранной зоны по периметру чувствительного элемента ПТП должна быть такой, чтобы обеспечить одномерность теплового потока в области размещения чувствительного элемента [4].

Анализ методических погрешностей, обусловленных нарушением одномерности температурного поля и поля теплового потока, обусловленные установкой ПТП на контролируемом объекте, выполнен путем математического моделирования температурного и теплового полей для наиболее типичных случаев применения ПТП на основании решения известной задачи теплопроводности в цилиндрических координатах с соответствующими граничными условиями. 
Распространенной является задача измерения плотности теплового потока при расположении ПТП внутри неограниченного массива (рис. $1, a)$. К таким объектам контроля относятся дисперсные засыпки, грунты, большие слои теплоизоляционных покрытий и др. Определяющими факторами для данной задачи являются соотношение геометрических размеров ПТП и отношение теплопроводности ПТП к теплопроводности объекта контроля [4-5]. Причем, глубина искажений достигает от 2 до 4 приведенных радиусов $\mathrm{r} / \mathrm{r}_{\text {птп }}$ в зоне, прилегающей к ПТП, а в самом ПТП искажения распространяются на глубину до пяти толщин ПТП. По результатам
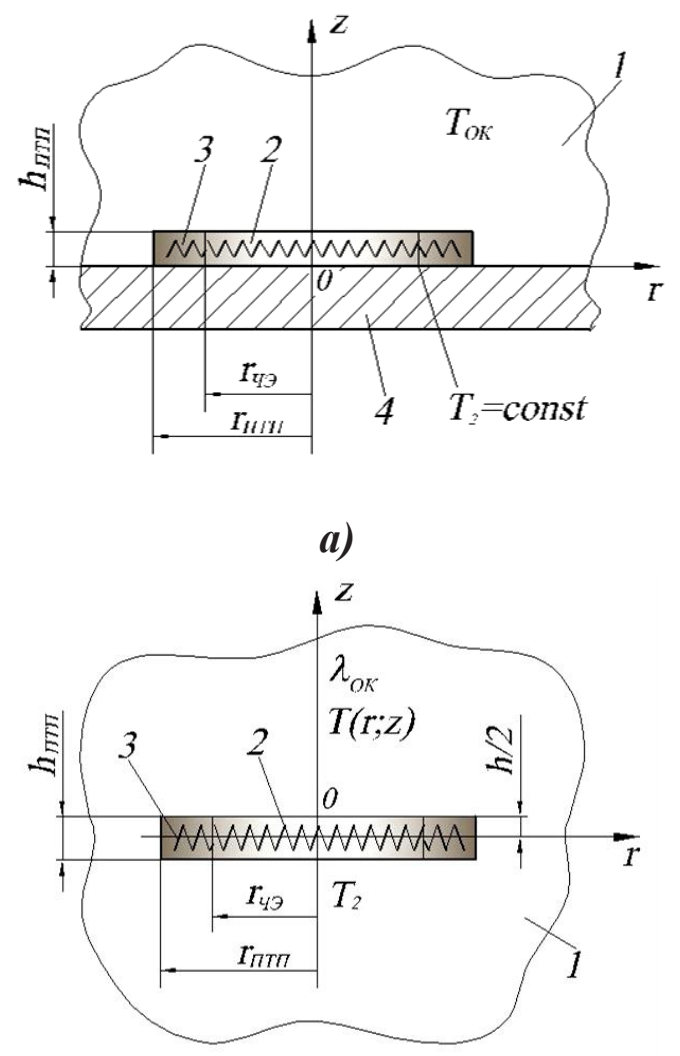

б) математического моделирования такой задачи построена номограмма (рис. 1, в) для выбора параметров ПТП или введения поправки в результат измерения.

Случаю расположения ПТП на изотермической поверхности, примыкающей к полуограниченному массиву (рис. 1, б), соответствует практическая ситуация измерения теплового потока через тонкую металлическую конструкцию преобразователем, установленным на ней в слое теплоизоляции, например, на металлической стенке теплоэнергетического оборудования под слоем мягкой теплоизоляции толщиной более пяти радиусов ПТП.

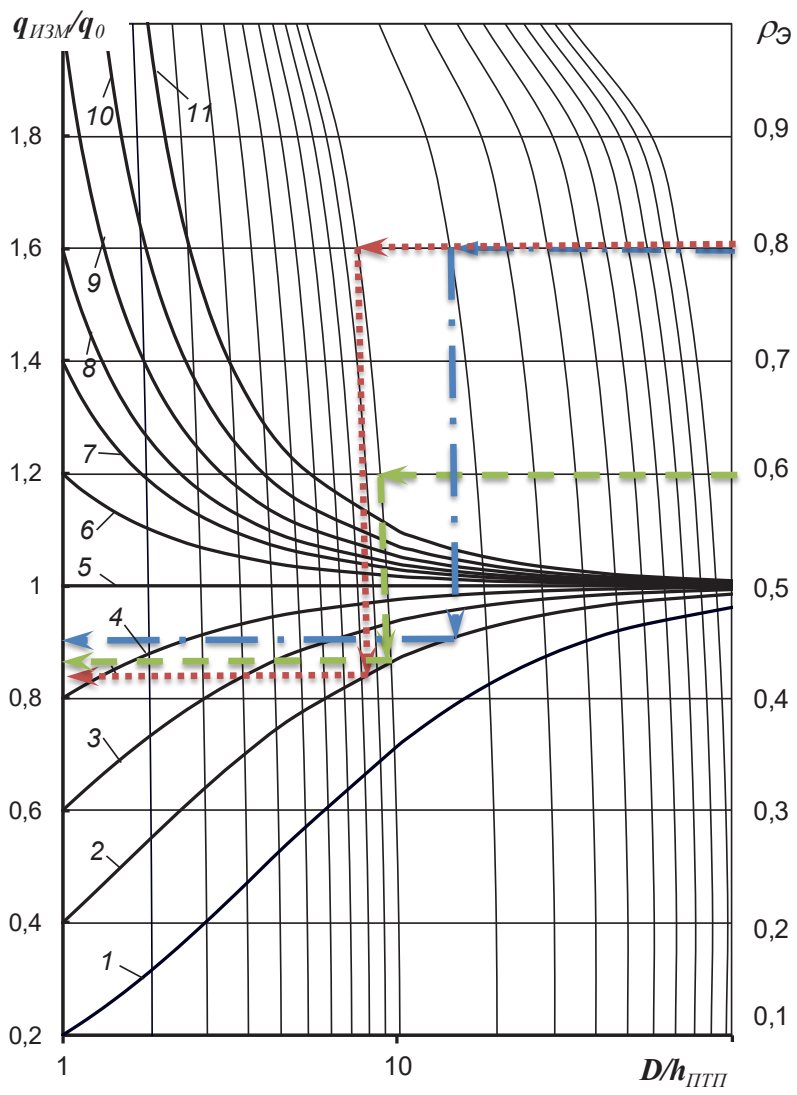

в) $1-\lambda_{\text {Птा }} / \lambda_{\text {OК }}=0,2 ; 2-0,4 ; \ldots ; 9-2,0$; $10-3,0 ; 11-10$.

Рис. 1. Схемы расположения ПТП внутри массива (a, б) и номограмма (в) зависимостей отночения значений плотности теплового потока от геометрического параметра $D / h_{\text {Iтгр }}$ величины приведенного радиуса чувствительного элемента $\rho_{\text {чЭ }}=r_{\text {чЭ }} / r_{\text {птп }} u$ относительной теплопроводности $\lambda_{\text {птті }} \lambda_{\text {ок }}$

а), б): 1 - объект контроля; 2 - чувствительный элемент ПТП;

3 - охранная зона ПТП; 4 - пластина металлическая. 
Номограмма, приведенная на рисунке 1, в применима и для этого случая, но при расчете приведенного радиуса используют удвоенную толщину ПТП ( $\left.\rho=\mathrm{r}_{\text {птп }} / 2 h_{\text {Птп }}\right)$.УМеньшить методическую погрешность можно за счет выравнивания теплофизических характеристик ПТП и объекта контроля, увеличения соотношения геометрических размеров ПТП $\left(D / h_{\Pi т \Pi}\right)$, введением охранной зоны в конструкцию ПТП.

При расположении ПТП на поверхности полуограниченного массива при граничных условиях 3-го рода (рис. 2, a), определяющими факторами влияния на величину методической погрешности являются: коэффициент теплообмена $\alpha$, геометрический размер ПТП $\mathrm{r}_{\text {птп }}$, теплопроводность объекта контроля $\lambda_{\text {ок }}$ и тепловое сопротивление ПТП $R_{\text {Птп, что учи- }}$ тывается приведенным тепловым сопротивлением $R_{\alpha, \text { оК }} /\left(R_{\alpha, \Pi т \Pi}+R_{\text {птп }}\right)$ и критерием Био $B i_{0, \mathrm{r}}=\alpha_{\mathrm{OK}} \cdot \mathrm{r}_{\Pi \pi \Pi} / \lambda_{\mathrm{OK}}$ (рис. 2, б) $[4,6]$. Методическая погрешность уменьшается при уменьшении

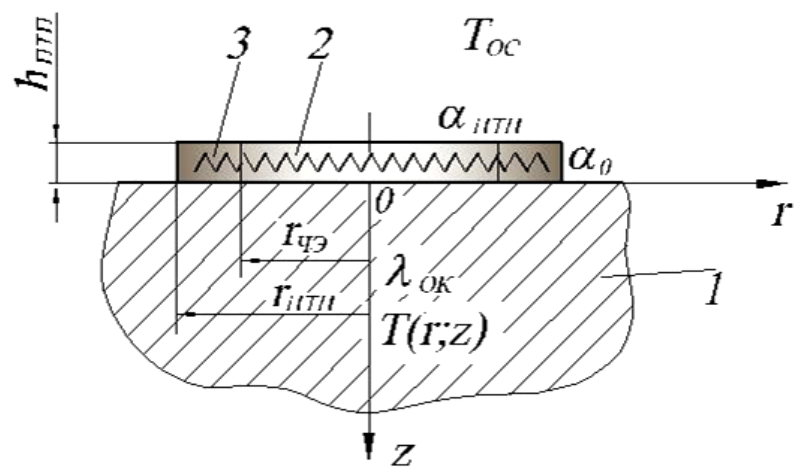

a)

1 - объект контроля,

2 - чувствительный элемент ПТП,

3 - охранная зона ПТП теплового сопротивления ПТП и увеличении его геометрического размера. Причем, с понижением теплопроводности объекта контроля влияние теплового сопротивления ПТП уменьшается.

Распространенным случаем измерения теплового потока является расположение ПТП на поверхности пластины со стороны конвективного теплообмена (граничные условия 3-го рода) при условии, что с другой стороны пластины теплообмен осуществляется при граничных условиях 2-го рода (рис. 3, a) [4, 7]. Установлено, что с ростом приведенной толщины $h_{\mathrm{OK}} / \mathrm{r}_{\text {Птп }}$ пластины методическая погрешность увеличивается (рис. 3,6 ). В случае, когда $h_{\text {оК }} / \mathrm{r}_{\text {птп }} \geq 1$ при определении методической погрешности допускается использование номограммы для полуограниченного массива (см. рис. 2, б). Выводы, сделанные для полуогрниченного массива, применимы и для данной задачи.

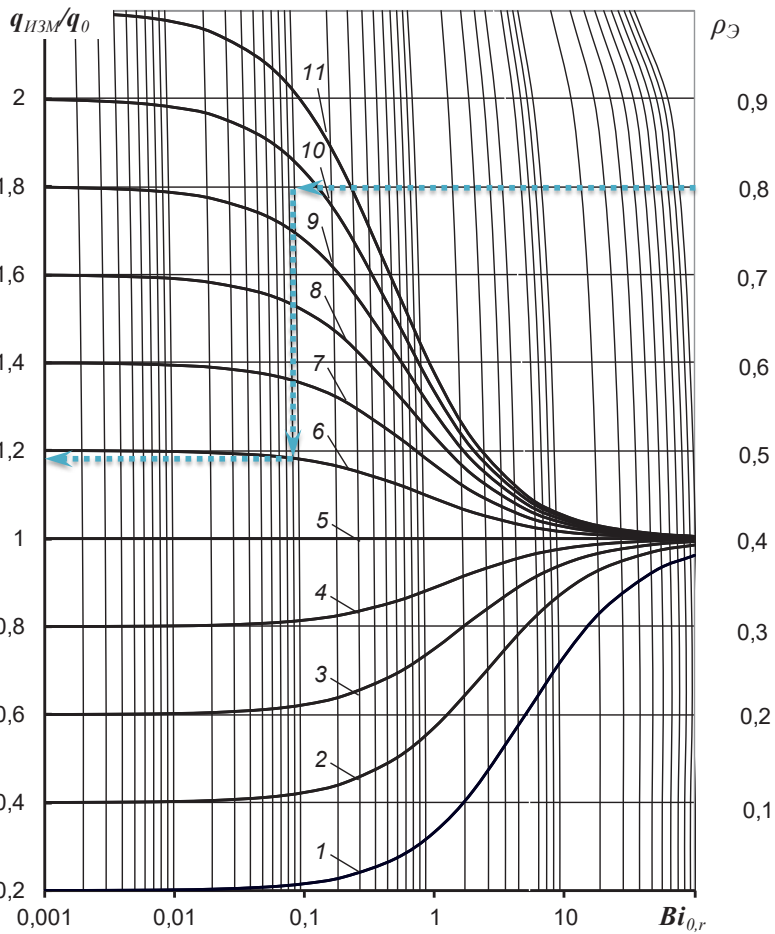

б) $1-\mathrm{R}_{\alpha, \text { ОК }} /\left(\mathrm{R}_{\alpha, \Pi \mathrm{T \Pi}}+\mathrm{R}_{\text {ПтП }}\right)=0,2 ; 2-0,4 ; \ldots$; $8-1,6 ; 9-1,8 ; 10-2,0 ; 11-2,2$.

Рис. 2. Схема расположения ПТП на поверхности полуограниченного массива (а) и номограмма для определения отночения значений плотности теплового потока (б). 


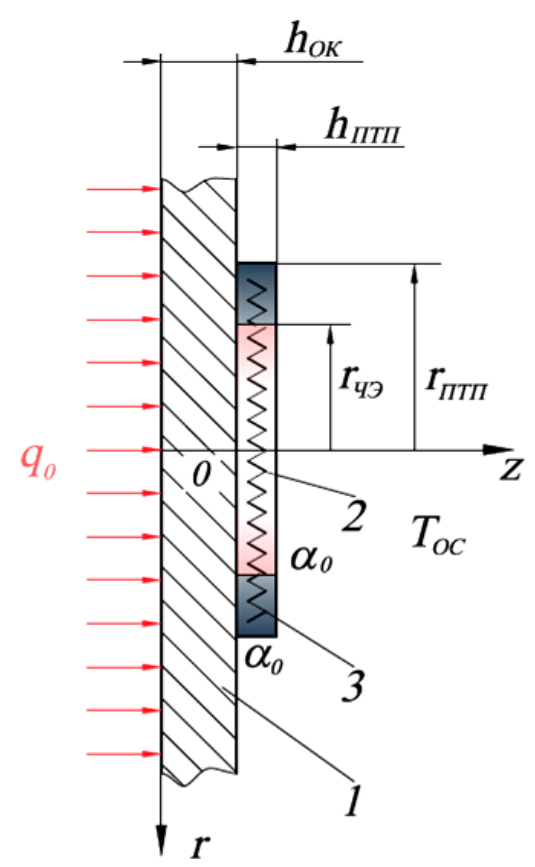

a)

1 - объект контроля,

2 - чувствительный элемент ПТП, 3 - охранная зона ПТП

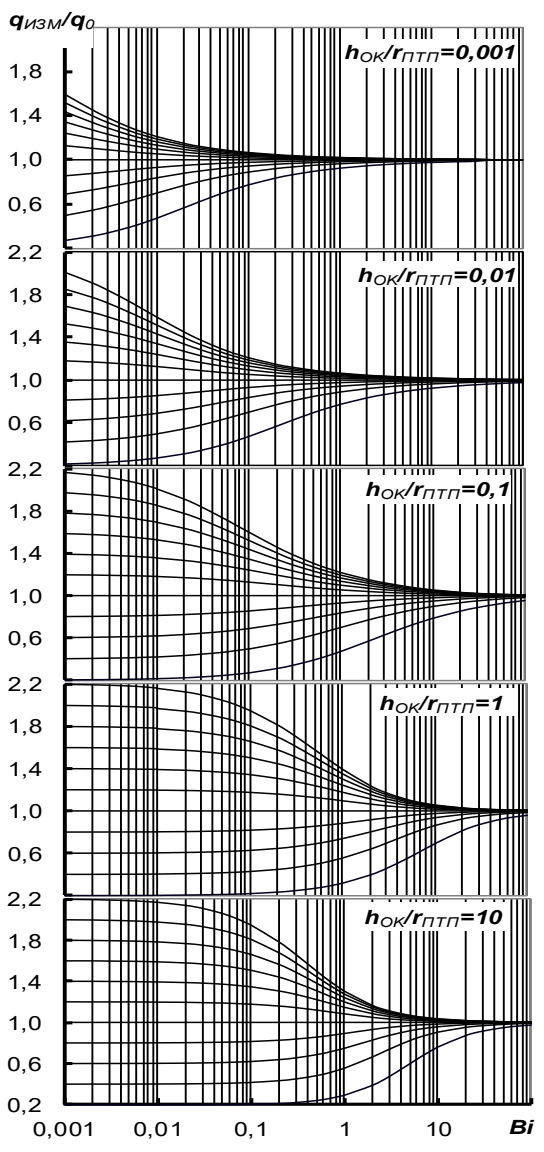

б)

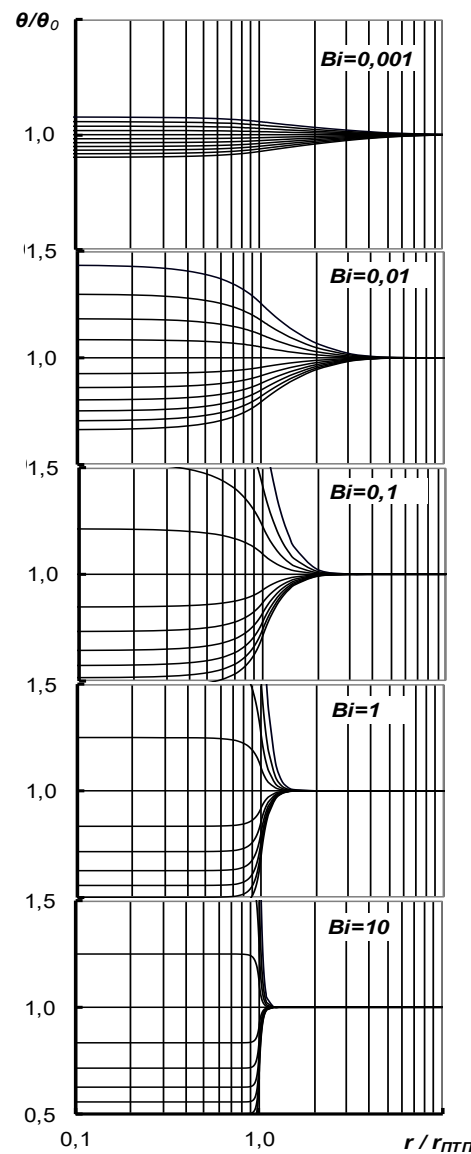

B)

Рис. 3. Схема расположения ПТП на поверхности неограниченной пластины (a) и зависимость отношения значений плотности теплового потока при вариации приведенной толщины (б) и критерия Био (в).

На рис. 3, в приведены результаты математического моделирования искажений температурного поля на поверхности пластины при вариации числовых значений критерия Био. Из представленных графиков следует, что с увеличением значений критерия Био возмущения температурного поля возрастают, но распространение искажений по поверхности объекта контроля локализуются в зоне расположения ПТП. Эта информация необходима для выбора мест установки поверхностных преобразователей разного назначения в невозмущенной зоне либо же для решения вопроса о расположении нескольких ПТП на одной поверхности без их взаимного влияния.

Наиболее востребованным является случай расположения ПТП на поверхности неограниченной пластины при конвективном теплообме- не с обеих сторон (рис. 4, a), описывающий теплометрические измерения при сертификации, стандартизации и паспортизации зданий и сооружений [4, 8-9]. Из представленных на рисунке $4, \sigma$ семейства графиков видно, что при приведенной толщине $h_{\text {ОК }} / \mathrm{r}_{\text {птп }} \geq 1$ искажения теплового потока не зависят от соотношения $\alpha_{2} / \alpha_{1}$ и аналогичны случаю расположения ПТП на полубесконечном массиве. Причем, с ростом $\alpha_{2}$ методическая погрешность возрастает. Остальные выводы совпадают с таковыми для предыдущей задачи.

\section{Выводы}

На основании представленных результатов с целью повышения точности измерения поверхностной плотности теплового потока сформулированы следующие рекомендации: 
1. В случае расположения ПТП внутри неограниченного массива либо на изотермической поверхности, примыкающей к полуограниченному массиву (см. рис. 1) целесообразно добиваться равенства теплопроводностей ПТП и объекта контроля.

2. При установке ПТП на поверхности объекта контроля (см. рис. 2-4) при конвективном и сложном теплообмене влияние на тепловое поле оказывает не общее сопротивление теплопередаче, а сопротивление теплообмену со стороны установки ПТП и тепловое сопротивление самого ПТП. Минимизации методической погрешности достигают снижением теплового сопротивления ПТП и контактного теплового сопротивления.

3. При сложном (конвективно-радиационном) теплообмене (см. рис. 4) кроме минимизации теплового сопротивления ПТП необходимо стремиться к выравниванию терморадиационных характеристик поверхностей ПТП и объекта контроля.

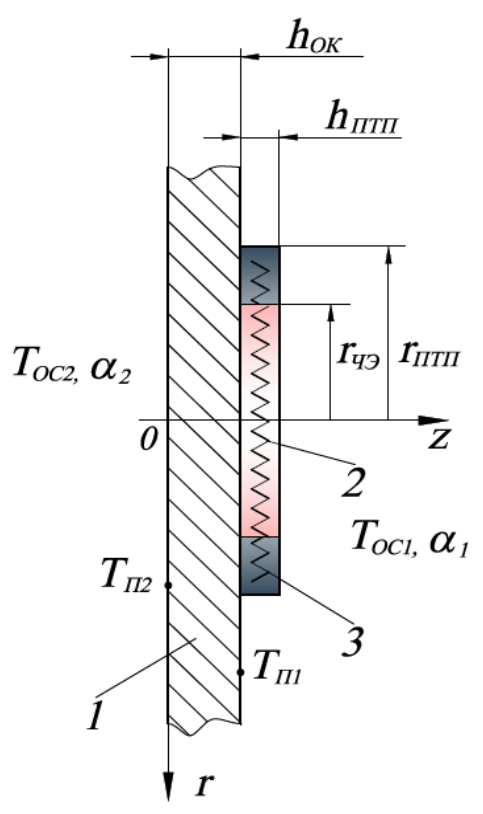

a)

1 - объект контроля,

2 - чувствительный элемент ПТП, 3 - охранная зона

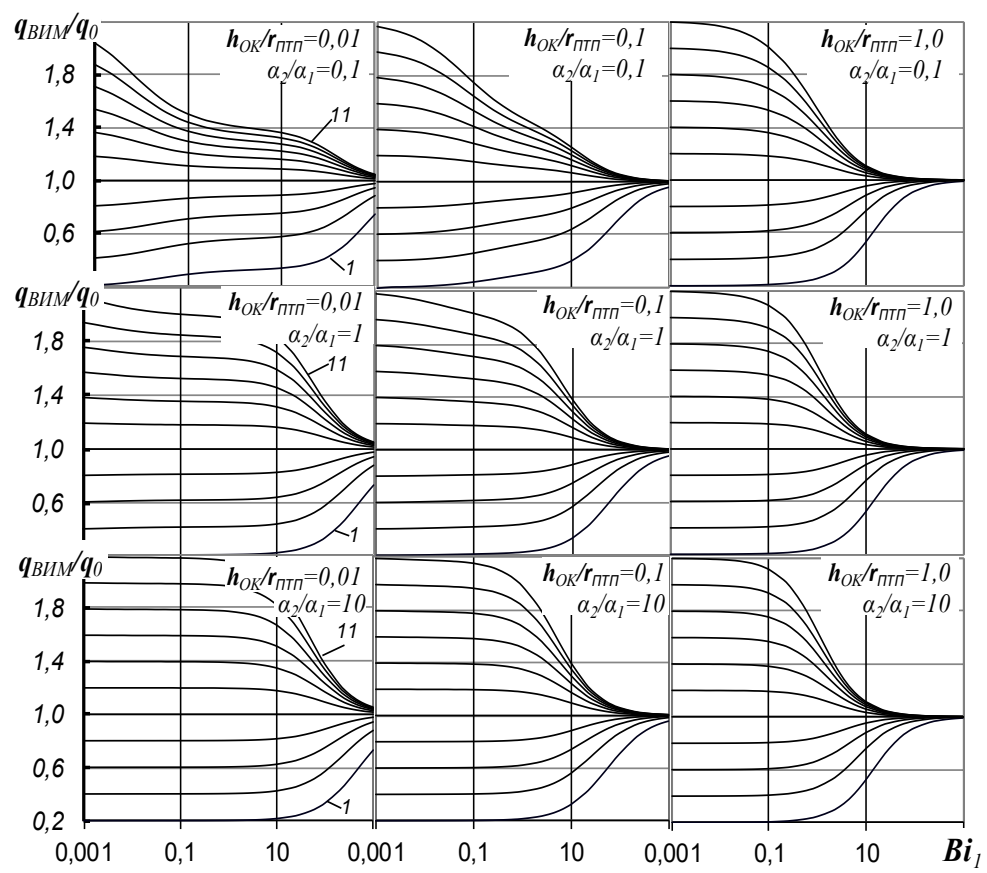

б) .

Рис. 4. Схема расположения ПТП на поверхности неограниченной пластины (a) и зависимость отночения значений плотности теплового потока от числа Био (б).

ЛИТЕРАТУРА

1. Енергозбереження. Методи та засоби вимірювання теплових величин. Загальні положення: ДСТУ 3401-97 (ГОСТ 30486-97). - [Чинний від 1999-01-01]. - К.: Держспоживстандарт України, 1998.-26с.

2. Теплоізоляиія. Будівельні елементи. Натурні вимірювання теплового опору та коефіцієнта теплопередавання (ISO 9869:1994, IDT): ДСТУ ISO 9869:2007. - [Чинний від 2009-01-01]. - К.: Держспоживстандарт України, 2007. - 46 с.
3. Теплоізоляція. Визначення теплового опору та пов'язаних із ним характеристик в усталеному режимі приладом із перетворювачем теплового потоку (ISO 8301:1991, IDT): ДСТУ ISO 8301:2007. - [Чинний від 2009-01-01].- К.: Держстандарт України, 2011. - 40с.

4. Визначення теплових потоків крізь огороджувальні конструкції: Методика М 00013184.5.023-01 / Розробники: Грищенко Т.Г. [та ін.] // Нормативний документ Комітету 3 будівництва та архітектури України. - К.: ЛО- 
ГОС, 2002. - $131 \mathrm{c}$.

5. Ярышев Н.А., Заровная Н.Н., Смирнова T.B. Влияние теплопроводности и размеров тепломера на точность измерения теплового потока // Инженерно-физический журнал. - 1988. - Т.55, №5. - C. 847-853.

6. Ярышев Н.А., Заровная Н.Н., Смирнова Т.В. Погрешность измерения стационарного теплового потока на поверхности тела // Инженерно-физический журнал. - 1989. - Т. 57, №4. - С. 667674.

7. Декуша Л.В., Гришенко Т.Г., Менделеева Т.В. Измерение стационарного теплового потока плоским преобразователем теплового потока, помещенным на конвективно охлаждаемой бес- конечной пластине // Деп. в ГНТБ Украины, р. № 39-Ук2003 - Киев, 2002. - 16 с.

8. Декуша Л.В., Менделеева Т.В. Об особенностях измерения теплового потока, проходящего через плоскую ограждающую конструкцию // Проблемы экологии и эксплуатации объектов энергетики (Севастополь-2002) : труды XII конф. СНГ с международным участием. - К.: ИПЦ «АЛКОН» НАУ, 2002. - С. 80-83.

9. Декуша Л.В., Менделеева Т.В. Измерение стационарного теплового потока, приходящего через бесконечную пластину по обе стороны, которой происходит конвективный теплообмен // Промышленная теплотехника. - Киев, 2007. T. 29, № 2. - C. 88-96. - ISSN 0204-3602. 


\section{IMPROVE ACCURACY OF HEAT FLUX DENSITY MEASUREMENT AT THE HEAT LOSSES CONTROL}

\section{Kovtun S.I.}

Institute of Engineering Thermophysics National Academy of Sciences of Ukraine, vul. Zhelyabova, 2a, Kiev, 03680, Ukraine

Key words: heat flow density, control of heat losses, measurement accuracy.

The objective. Determination of conditions conducive to improving the accuracy of measurement of the heat flux density in determining the heat losses on various objects-to-be-controlled with using of thermoelectric heat flux transducers of auxiliary wall type.

Results. At the control of heat losses one of the defining parameters is the surface heat flux density. Heat flux transducers have their own thermal resistance, so their location on the object-to-be-controlled perturbs the field of temperature and heat flow within the object, and in the transducer, which certainly leads to a decrease in the measurement accuracy. In order to ensure the required accuracy of measurement of the surface heat flux density at inspection of the concrete object-to-be-controlled should be used transducers with the appropriate parameters: dimensions, aspect transducer and sensing element ratio, wide the protected zone, thermal properties, which will be provided at a minimum methodical error. In the work the methodological errors were analyzed as the largest component of the measurement results error for a few typical applications heat flux transducers to control different types of objects under different conditions of heat exchange. From the obtained results recommendations are formulated.

References 9, figures 4 .

1. Energy-saving. Methods and tools for measuring thermal quantities. General provisions:
ДСТУ 3401-97 (ГОСТ 30486-97). - [Valid from 1999-01-01]. Derghspoghivstandart, 1998. - 24p. (Ukr.)

2. Thermal insulation. Building elements. In-situ measurement of thermal resistance and thermal transmittance (ISO 9869:1994, IDT): ДСТУ ISO 9869:2007. - [Valid from 2009-01-01]. Derghspoghivstandart, 2007. - 46p. (Ukr.)

3. Thermal insulation. Determination of steadystate thermal resistance and related properties. Heat flow meter apparatus (ISO 8301:1991, IDT): ДСТУ ISO 8301:2007. - [Valid from 2009-01-01].Derghstandart, 2011. - 40 p. (Ukr.)

4. Grischenko, T. \& others (2002). Determination of the heat flow through the walling: Methodology M 00013184.5.023-01. Regulation of the Committee of Construction and Architecture of Ukraine. - Kyiv: LOGOS (ISBN 966-581-354-4), 131p. (Ukr./Rus.)

5. Yaryshev, N. (1989). Influence of thermal conductivity and dimensions of heat flow meter to the accuracy of heat flow measurement. Journal of Engineering Physics, Vol. 55, №5, P. 847-853. (Rus.)

6 Yaryshev, N. (1989). Measuring error of the stationary heat flow on the body surface. Journal of Engineering Physics, Vol. 57, №4, P. 667-674. (Rus.)

7. Grischenko, T., Dekusha, L. and Mendeleyeva, T. (2002). Measurement of stationary thermal flow by flat heat flow transducer placed on an infinite plate convectively cooled, Д/п ГНТБ Ukraine, № 39-Ук2003. - 16 p. (Rus.)

8. Dekusha, L., \& Mendeleyeva, T. (2002). Specifics measuring the heat flow through the flat walling. Problems of Ecology and Operation Energy Objects (Sevastopol, 2002), P. 80-83. (Rus.)

9. Dekusha, L., \& Mendeleyeva, T. (2007). Measurement of stationary heat flow passing through an infinite plate on both sides which the convective heat transfer takes place. Industrial Heat Engineering, Vol.29 №2 (ISSN 0204-3602), P. 88-96. (Rus.)

Получено 25.11.2015 Received 25.11.2015 